\title{
The Fight Against Severe COVID-19: Can Parasitic Worms Contribute?
}

\author{
Pengfei Cai ${ }^{*}$, Yi Mu and Donald P. McManus* \\ Molecular Parasitology Laboratory, QIMR Berghofer Medical Research Institute, Brisbane, QLD, Australia
}

\section{OPEN ACCESS}

Edited by:

Tengchuan Jin,

University of Science and Technology

of China, China

Reviewed by:

David Gems,

University College London,

United Kingdom

Bruce Zhang,

University College London,

United Kingdom,

in collaboration with reviewer $D G$

Makedonka Mitreva,

Washington University School of

Medicine in St. Louis, United States

*Correspondence:

Pengfei Cai

Pengfei.Cai@gimrberghofer.edu.au.

Donald P. McManus

Don.McManus@

qimrberghofer.edu.au

Specialty section:

This article was submitted to

Viral Immunology,

a section of the journal

Frontiers in Immunology

Received: 06 January 2022

Accepted: 21 January 2022

Published: 11 February 2022

Citation:

Cai P, Mu Y and McManus DP (2022)

The Fight Against Severe COVID-19:

Can Parasitic Worms Contribute?

Front. Immunol. 13:849465.

doi: 10.3389/fimmu.2022.849465
Keywords: COVID-19, SARS-CoV-2, cytokine storm, viral sepsis, helminth, immunomodulatory therapy

\section{INTRODUCTION}

As of 31 December 2021, COVID-19, caused by infection with SARS-CoV-2, had been confirmed in more than 285 million people worldwide, with more than 5.4 million dead resulting in a case fatality ratio of $1.89 \%$. This figure is likely to be vastly underestimated, as a proportion was not registered officially as COVID-19-related/excess deaths. The United States recorded the highest number $(54,656,866)$ of confirmed cases. In Africa, there are 47 countries affected, with 7,065,972 cumulative cases and 155,081 deaths were recorded by 31 Dec 2021 (WHO African Region numbers at a glance). To date, the currently approved vaccines have been effective in preventing COVID-19, particularly in regards to severe symptoms (1). However, several immune escape mechanisms of SARS-CoV-2 and the rapid emergence of mutated variants (2) pose a great challenge to the efficacy of these vaccines.

Patients with severe COVID-19 tend to have a high concentration of pro-inflammatory cytokines (IL-2, IL-7, IL-10, G-CSF, TNF- $\alpha$, CXCL10, MCP1, and MIP1 $\alpha$ ) (3), suggesting that a cytokine release syndrome (CRS) (4) (also loosely referred to as a cytokine storm), which is a form of life-threatening systemic inflammatory response syndrome (SIRS), can often feature in severe COVID-19 infections. Among the increased levels of inflammatory mediators in COVID-19 patients, the plasma levels of IL-6, an amplifier in the cytokine storm, are significantly elevated in non-survivors compared with survivors (5). The main cause of death of COVID-19 is due to severe acute respiratory distress syndrome (ARDS) with this high severity being dependent on the cytokine storm.

Sepsis has been defined as a life-threatening organ dysfunction caused by a dysregulated host response to infection (6). Endothelium damage, vascular permeability, microvascular dysfunction, coagulation pathway activation, and impaired tissue oxygenation occur during sepsis and can lead to multisystem organ dysfunction (MODS), organ failure and consequently a potentially lethal outcome. As many patients with severe COVID-19 show typical clinical manifestations of septic shock, with other symptoms meeting the diagnostic criteria for sepsis and septic shock according to the Sepsis-3 International Consensus (6), Li et al. hypothesized that viral sepsis is a crucial process in severe COVID-19 cases (7). Accumulating evidence further links the pathology of severe COVID19 , such as acute kidney injury, to sepsis (8). 


\section{IMMUNOMODULATORY THERAPY OF SEVERE COVID-19}

In regards to potential immunomodulatory strategies for severe COVID-19, the IL-6-STAT3 signaling pathway has been considered a promising therapeutic target for the cytokine storm generated in the disease. Tocilizumab, a specific monoclonal antibody that blocks IL-6, has been recommended for use in critically ill COVID-19 patients with extensive bilateral pulmonary lesions and with elevated serum levels of IL-6. However, anticytokine therapy with Tocilizumab did not improve survival rates despite reducing the likelihood of progression to the composite outcome of mechanical ventilation or death (9). In addition, ulinastatin, a serine protease inhibitor with anti-inflammatory properties (including inhibition of IL-6), previously used in the treatment of acute pancreatitis and sepsis, has been suggested for severe COVID-19 treatment (10); yet its clinical performance and cost-effectiveness remain to be validated in large cohort studies.

The value of glucocorticoids in mitigating the inflammatory response due to COVID-19 has been widely scrutinized. Recent reliable evidence from large-scale randomized clinical trials (RCTs) revealed that the use of dexamethasone reduced 28-day mortality but only in patients requiring respiratory support (11), while another parallel, double-blind, placebo-controlled, randomized, Phase IIb clinical trial showed that the administration of methylprednisolone was able to reduce 28-day mortality in patients aged over 60 years (12). In addition, hydroxychloroquine, a disease-modifying antirheumatic drug (DMARD), used for the treatment of rheumatoid arthritis and lupus, has been studied for its potential as an immunomodulatory therapeutic for COVID-19 disease. Evidence from 12 RCTs indicated that hydroxychloroquine has little or no effect on the risk of death, probably has no effect on progression to mechanical ventilation, and that it is less likely that the drug is effective in protecting people from infection, although this was not excluded entirely (13). Other immunomodulatory agents that have been therapeutically tested in SARS-CoV-2 infection include the interleukin-1 receptor (IL-1R) antagonist anakinra, the Janus kinase inhibitors baricitinib and ruxolitinib, the anti-C5a antibody vilobelimab, the anti-gout agent colchicine, the antirheumatic drug leflunomide, convalescent plasma, interferon beta, interferon kappa and intravenous immunoglobulins (IVIg) (14). However, robust data from further RCTs are required to elucidate their potential for the treatment of severe COVID-19.

\section{HELMINTH CO-INFECTION AND SEVERITY OF COVID-19}

The "old friends" hypothesis argues that some co-evolved microbes and other pathogens, including helminths, could help to establish appropriate immunomodulatory function and thus protect the host against a large spectrum of immune-related disorders (15). Mammals infected with helminths typically elicit an anti-inflammatory Th2 immune response, including the activation of Th2 cells and the elevation of Th2-type cytokines such as IL-4, IL-5 and IL-13 (16). This host-helminth interaction could be beneficial in dampening inflammatory damage induced by the Th1/Th17 branches of the immune system, repairing injured tissue and restoring homeostasis (17). Chronic helminthic infection suppresses both Th1 and Th2 responses by actively inducing the expansion of $\mathrm{FOXP}^{3+}$ regulatory $\mathrm{T}$ cells, IL-10 producing $B$ cells and alternatively activated macrophages (AAMs), which together promote the release of regulatory cytokines such as TGF- $\beta$ and IL-10 (18).

There is controversy regarding whether helminth coinfection leads to increased susceptibility and attenuated immunopathology of other pathogens (i.e., viruses, bacteria and protozoa) or, in some circumstance, exacerbated pathology due to higher infection burdens (19). And this also likely applies to the interaction between helminths and SARS-CoV-2 (20, 21). It has been suggested that the immunosuppressive and regulatory $\mathrm{T}$-helper response stimulated by helminths may balance the inflammatory Th1/Th17 response triggered by SARS-CoV-2 infection, potentially restricting the severity of COVID-19 disease $(22,23)$. In contrast, a recent viewpoint article argued that COVID-19 patients co-infected with helminths may be unable to mount a quick and efficient immune response against SARS-CoV-2 in the early phase of the infection, thereby leading to increased patient morbidity and mortality (24). However, other evidence indicates that COVID-19 lethality rates are significantly lower in Sub-Saharan Africa than in the industrialized world (25). Wolday et al. (26) carried out a prospective observational cohort study to investigate whether there was a potential correlation between co-infection with intestinal parasites and the severity of COVID-19 in two sites in an endemic area of Ethiopia in Sub-Saharan Africa. The study revealed that patients co-infected with parasites had lower odds of developing severe COVID-19, with an adjusted odds ratio (aOR) of $0.23(p<0.0001)$ for all parasites, an aOR of $0.37(p<0.0001)$ for protozoa, and an aOR of $0.26(p<0.0001)$ for helminths. The authors thus concluded that co-infection with the enteric parasites, Hymenolopis nana, Schistosoma mansoni and Trichuris trichiura reduced the risk of severe COVID-19 occurrence in this cohort of African patients. When stratified by species, co-infection with $T$. trichiura showed the lowest probability of developing severe COVID-19. In addition, of 11 cohort patients who died, all were parasite-free (26). The results of this study thus suggested that parasites, particularly the chronic disease-associated parasitic helminths, induced a Th2-prone response in the host, which modulates COVID-19 severity by restricting the hyperinflammation associated with the viral infection. Further epidemiological studies on helminth-mediated COVID-19 alleviation are, however, required to support this argument $(27,28)$.

\section{HELMINTH-DERIVED PRODUCTS CAN ATTENUATE THE SEVERITY OF SEPSIS}

The "old friends" hypothesis, together with the inverse global distribution of allergy/autoimmune diseases and helminth infections, and the proclivity for helminths to orchestrate immunomodulatory effects (typically induction of a Th2 
immune response) on the host immune system stimulated the concept of developing helminth-based therapies. Robust evidence from animal model studies showed that helminth infection and helminth-derived products were able to prevent/ alleviate a variety of autoimmune and inflammatory diseases/ disorders (i.e., sepsis, type 2 diabetes, allergic asthma, rheumatoid arthritis, inflammatory bowel disease, type 1 diabetes and multiple sclerosis) (29, 30).

In regard to sepsis, epidemiological studies (over the period 2006-2015) indicated a rapid increase in hospitalization and mortality rates due to severe sepsis in high-income countries (31). This report added further support to the hypothesis that the lack of helminth infections may contribute to the aetiology of sepsis (32). To date, a number of helminth-derived molecular products have resulted in improved sepsis outcomes in animal models. Several studies have investigated the role of Schistosoma japonicum cystatin ( $\mathrm{rSj}$-Cys) in regulating the inflammatory response in the cecal ligation and puncture (CLP)-induced mouse sepsis model (33-35). Administration of rSj-Cys to mice provided significant therapeutic effects on CLP-induced sepsis characterized by increased survival rates, alleviated overall disease severity with reduced tissue injury in the kidney, lung and liver (33) and cardiomyopathy (34). These therapeutic effects were linked to the upregulation of regulatory cytokines (IL-10 and TGF- $\beta 1$ ) and the downregulation of pro-inflammatory cytokines (IL-1 $\beta$, IL-6 and TNF- $\alpha$ ) as measured in serum. Similarly, treatment of mice with cyclophilin A (CsCyPA) from the liver fluke, Clonorchis sinensis, provided significant therapeutic effects on CLP-induced sepsis characterized by an improved survival rate (36). Furthermore, using a murine model of septic shock, RamosBenitez et al. demonstrated in vitro and in vivo that Fh15, a recombinant variant of the common liver fluke Fasciola hepatica fatty acid binding protein, suppressed the LPS-induced cytokine storm, working as an antagonist of Toll-like receptor 4 (TLR4) (37). In the gram-negative bacteria-induced sepsis rhesus macaque model, Fh15 effectively suppressed bacteremia, endotoxemia, and many other inflammatory markers, emphasizing its promise as a candidate for immunomodulatory therapy against sepsis (38). In addition, the excretory-secretory products of Trichinella spiralis adult worms were also shown to be beneficial to the outcome of CLP-induced sepsis by preventing exacerbated inflammation and severe pathology in treated mice (39). These effects were associated with reduced levels of pro-inflammatory cytokines (IL-1 $\beta$, IL- 6 and TNF- $\alpha$ ), upregulated levels of IL-10 and TGF- $\beta$, and decreased expression of HMGB1, TLR2 and MyD88 in the lung tissues of the treated mice (39). Albeit informative, these observations were accrued from animal sepsis models and human clinical trials now need to be undertaken to validate the results obtained.

\section{DISCUSSION}

Rapid mutations in SARS-CoV-2 challenge the efficacy of the current COVID-19 vaccines and concerns about their long-term safety require an urgent need to search for safe and cost-effective alternatives for preventing severe COVID-19 disease. Increased efforts are required as, to date, conclusive evidence of effective immunomodulatory therapies for severe COVID-19 is scarce (14). Due to similarities in the pathological process, sepsis animal models provide the opportunity to evaluate the efficacy of novel candidates for the immunomodulatory therapy of critically ill COVID-19 patients. We argue that helminth-derived products and molecules that can potentially induce a Th2-biased immune response may provide a contributory role in preventing severe COVID-19 by restricting the cytokine storm associated with ARDS. The aforementioned helminth-derived molecules (i.e. rSj-Cys, CsCyPA and Fh15) have been shown to increase survival rates in animal models of sepsis, thereby representing potential candidates for immunomodulatory treatment against severe COVID-19. Such components should be validated for efficacy, first in the K18hACE2 transgenic murine model of SARS-CoV-2 infection which shares many features of severe COVID-19 infection (40), and then in clinical cohorts. The time phase in sepsis progression is regarded as a key factor for successful immunomodulatory therapy. Due to immunosuppression and immune exhaustion, treatment with immunomodulators at the late stage of severe COVID-19 could be less effective or even deleterious; consequently, the helminthderived molecular products should be administrated as a prophylactic therapy against severe COVID-19. The suppression of the antiviral response due to excessive immunotherapy may encourage viral replication and result in a delay of clearance of SARS-CoV-2 so that administration of helminth-derived immunomodulators that elicit a mild Th2-skewed immune response could be a useful strategy to prevent severe COVID-19, while maintaining the patient's ability to kill cells infected with the virus. Severe COVID-19 has greater incidence in older individuals, due in part to an increased inflammatory response in these patients (41), begging the question whether prophylactic therapy based on helminth-derived product should primarily target and would be more effective in older individuals? Another unanswered question is whether a well-controlled low level concomitant infection with a live helminth, such as hookworm (42), can achieve an equivalent or superior effect to an immunomodulator or vaccine in preventing serious outcomes of COVID-19. It would be valuable to test such helminthic-based therapies as these may represent a safe and costeffective anti-inflammation approach to reducing COVID19 severity.

\section{AUTHOR CONTRIBUTIONS}

Conceptualization: PC, YM, and DM. Writing - original draft: PC and YM. Writing - review and editing: PC and DM. All authors contributed to the article and approved the submitted version.

\section{FUNDING}

This work was partly supported by the National Health and Medical Research Council (NHMRC) of Australia (ID: APP1160046, APP2008433 and APP1098244). DPM is a NHMRC Leadership Fellow and Senior Scientist at QIMRB. 


\section{REFERENCES}

1. Tregoning JS, Flight KE, Higham SL, Wang Z, Pierce BF. Progress of the COVID-19 Vaccine Effort: Viruses, Vaccines and Variants Versus Efficacy, Effectiveness and Escape. Nat Rev Immunol (2021) 21(10):626-36. doi: 10.1038/s41577-021-00592-1

2. Harvey WT, Carabelli AM, Jackson B, Gupta RK, Thomson EC, Harrison EM, et al. SARS-CoV-2 Variants, Spike Mutations and Immune Escape. Nat Rev Microbiol (2021) 19(7):409-24. doi: 10.1038/s41579-021-00573-0

3. Huang C, Wang Y, Li X, Ren L, Zhao J, Hu Y, et al. Clinical Features of Patients Infected With 2019 Novel Coronavirus in Wuhan, China. Lancet (2020) 395(10223):497-506. doi: 10.1016/S0140-6736(20)30183-5

4. Moore JB, June CH. Cytokine Release Syndrome in Severe COVID-19. Science (2020) 368(6490):473-4. doi: 10.1126/science.abb8925

5. Hojyo S, Uchida M, Tanaka K, Hasebe R, Tanaka Y, Murakami M, et al. How COVID-19 Induces Cytokine Storm With High Mortality. Inflamm Regen (2020) 40:37-7. doi: 10.1186/s41232-020-00146-3

6. Singer M, Deutschman CS, Seymour CW, Shankar-Hari M, Annane D, Bauer $\mathrm{M}$, et al. The Third International Consensus Definitions for Sepsis and Septic Shock (Sepsis-3). JAMA (2016) 315(8):801-10. doi: 10.1001/jama.2016.0287

7. Li H, Liu L, Zhang D, Xu J, Dai H, Tang N, et al. SARS-CoV-2 and Viral Sepsis: Observations and Hypotheses. Lancet (2020) 395(10235):1517-20. doi: 10.1016/S0140-6736(20)30920-X

8. Alexander MP, Mangalaparthi KK, Madugundu AK, Moyer AM, Adam BA, Mengel M, et al. Acute Kidney Injury in Severe COVID-19 Has Similarities to Sepsis-Associated Kidney Injury: A Multi-Omics Study. Mayo Clin Proc (2021) 96(10):2561-75. doi: 10.1016/j.mayocp.2021.07.001

9. Salama C, Han J, Yau L, Reiss WG, Kramer B, Neidhart JD, et al. Tocilizumab in Patients Hospitalized With Covid-19 Pneumonia. N Engl J Med (2020) 384 (1):20-30. doi: 10.1056/NEJMoa2030340

10. Lin HY. The Severe COVID-19: A Sepsis Induced by Viral Infection? And Its Immunomodulatory Therapy. Chin J Traumatol (2020) 23(4):190-5. doi: $10.1016 /$ j.cjtee.2020.06.002

11. Group RC, Horby P, Lim WS, Emberson JR, Mafham M, Bell JL, et al. Dexamethasone in Hospitalized Patients With Covid-19. N Engl J Med (2021) 384(8):693-704. doi: 10.1056/NEJMoa2021436

12. Jeronimo CMP, Farias MEL, Val FFA, Sampaio VS, Alexandre MAA, Melo GC, et al. Methylprednisolone as Adjunctive Therapy for Patients Hospitalized With Coronavirus Disease 2019 (COVID-19; Metcovid): A Randomized, Double-Blind, Phase IIb, Placebo-Controlled Trial. Clin Infect Dis (2021) 72(9):e373-81. doi: 10.1093/cid/ciaal177

13. Singh B, Ryan H, Kredo T, Chaplin M, Fletcher T. Chloroquine or Hydroxychloroquine for Prevention and Treatment of COVID-19. Cochrane Database Syst Rev (2021) 2:CD013587. doi: 10.1002/14651858. CD013587.pub2

14. Alunno A, Najm A, Mariette X, De Marco G, Emmel J, Mason L, et al. Immunomodulatory Therapies for SARS-CoV-2 Infection: A Systematic Literature Review to Inform EULAR Points to Consider. Ann Rheum Dis (2021) 80(6):803-15. doi: 10.1136/annrheumdis-2020-219725

15. Rook GA, Adams V, Hunt J, Palmer R, Martinelli R, Brunet LR. Mycobacteria and Other Environmental Organisms as Immunomodulators for Immunoregulatory Disorders. Springer Semin Immunopathol (2004) 25(34):237-55. doi: 10.1007/s00281-003-0148-9

16. Harris NL, Loke P. Recent Advances in Type-2-Cell-Mediated Immunity: Insights From Helminth Infection. Immunity (2017) 47(6):1024-36. doi: 10.1016/j.immuni.2017.11.015

17. Allen JE, Maizels RM. Diversity and Dialogue in Immunity to Helminths. Nat Rev Immunol (2011) 11(6):375-88. doi: 10.1038/nri2992

18. Turner JD, Jackson JA, Faulkner H, Behnke J, Else KJ, Kamgno J, et al. Intensity of Intestinal Infection With Multiple Worm Species Is Related to Regulatory Cytokine Output and Immune Hyporesponsiveness. J Infect Dis (2008) 197(8):1204-12. doi: 10.1086/586717

19. McSorley HJ, Maizels RM. Helminth Infections and Host Immune Regulation. Clin Microbiol Rev (2012) 25(4):585-608. doi: 10.1128/CMR. 05040-11

20. Ademe M, Girma F. The Influence of Helminth Immune Regulation on COVID-19 Clinical Outcomes: Is It Beneficial or Detrimental? Infect Drug Resist (2021) 14:4421-6. doi: 10.2147/idr.S335447
21. Głuchowska K, Dzieciątkowski T, Sędzikowska A, Zawistowska-Deniziak A, Młocicki D. The New Status of Parasitic Diseases in the COVID-19 Pandemic-Risk Factors or Protective Agents? J Clin Med (2021) 10 (11):2533. doi: $10.3390 / \mathrm{jcm} 10112533$

22. Cepon-Robins TJ, Gildner TE. Old Friends Meet a New Foe: A Potential Role for Immune-Priming Parasites in Mitigating COVID-19 Morbidity and Mortality. Evol Med Public Health (2020) 2020(1):234-48. doi: 10.1093/ emph/eoaa037

23. Hays R, Pierce D, Giacomin P, Loukas A, Bourke P, McDermott R. Helminth Coinfection and COVID-19: An Alternate Hypothesis. PloS Negl Trop Dis (2020) 14(8):e0008628. doi: 10.1371/journal.pntd.0008628

24. Abdoli A. Helminths and COVID-19 Co-Infections: A Neglected Critical Challenge. ACS Pharmacol Transl Sci (2020) 3(5):1039-41. doi: 10.1021/ acsptsci.0c00141

25. Fonte L, Acosta A, Sarmiento ME, Ginori M, Garcia G, Norazmi MN. COVID-19 Lethality in Sub-Saharan Africa and Helminth Immune Modulation. Front Immunol (2020) 11:574910. doi: 10.3389/fimmu. 2020.574910

26. Wolday D, Gebrecherkos T, Arefaine ZG, Kiros YK, Gebreegzabher A, Tasew G, et al. Effect of Co-Infection With Intestinal Parasites on COVID-19 Severity: A Prospective Observational Cohort Study. EClinicalMedicine (2021) 39:101054. doi: 10.1016/j.eclinm.2021.101054

27. Bradbury RS, Piedrafita D, Greenhill A, Mahanty S. Will Helminth CoInfection Modulate COVID-19 Severity in Endemic Regions? Nat Rev Immunol (2020) 20(6):342. doi: 10.1038/s41577-020-0330-5

28. Siles-Lucas M, Gonzalez-Miguel J, Geller R, Sanjuan R, Perez-Arevalo J, Martinez-Moreno A. Potential Influence of Helminth Molecules on COVID-19 Pathology. Trends Parasitol (2021) 37(1):11-4. doi: 10.1016/ j.pt.2020.10.002

29. Smallwood TB, Giacomin PR, Loukas A, Mulvenna JP, Clark RJ, Miles JJ. Helminth Immunomodulation in Autoimmune Disease. Front Immunol (2017) 8:453. doi: 10.3389/fimmu.2017.00453

30. Mu Y, McManus DP, Hou N, Cai P. Schistosome Infection and SchistosomeDerived Products as Modulators for the Prevention and Alleviation of Immunological Disorders. Front Immunol (2021) 12:619776. doi: 10.3389/ fimmu.2021.619776

31. Fleischmann C, Scherag A, Adhikari NK, Hartog CS, Tsaganos T, Schlattmann P, et al. Assessment of Global Incidence and Mortality of Hospital-Treated Sepsis. Current Estimates and Limitations. Am J Respir Crit Care Med (2016) 193(3):259-72. doi: 10.1164/rccm.201504-0781OC

32. Hubner MP, Layland LE, Hoerauf A. Helminths and Their Implication in Sepsis - A New Branch of Their Immunomodulatory Behaviour? Pathog Dis (2013) 69(2):127-41. doi: 10.1111/2049-632X.12080

33. Li H, Wang S, Zhan B, He W, Chu L, Qiu D, et al. Therapeutic Effect of Schistosoma Japonicum Cystatin on Bacterial Sepsis in Mice. Parasit Vectors (2017) 10(1):222. doi: 10.1186/s13071-017-2162-0

34. Gao S, Li H, Xie H, Wu S, Yuan Y, Chu L, et al. Therapeutic Efficacy of Schistosoma Japonicum Cystatin on Sepsis-Induced Cardiomyopathy in a Mouse Model. Parasit Vectors (2020) 13(1):260. doi: 10.1186/s13071-02004104-3

35. Xie H, Wu L, Chen X, Gao S, Li H, Yuan Y, et al. Schistosoma Japonicum Cystatin Alleviates Sepsis Through Activating Regulatory Macrophages. Front Cell Infect Microbiol (2021) 11:617461. doi: 10.3389/fcimb.2021.617461

36. Jiang J, Yin H, Sun Y, Huang H, Hu X. Clonorchis Sinensis Cyclophilin A Immunization Protected Mice From CLP-Induced Sepsis. Int Immunopharmacol (2018) 59:347-53. doi: 10.1016/j.intimp.2018.03.039

37. Ramos-Benitez MJ, Ruiz-Jimenez C, Rosado-Franco JJ, Ramos-Pérez WD, Mendez LB, Osuna A, et al. Fh15 Blocks the Lipopolysaccharide-Induced Cytokine Storm While Modulating Peritoneal Macrophage Migration and CD38 Expression Within Spleen Macrophages in a Mouse Model of Septic Shock. mSphere (2018) 3(6):e00548-00518. doi: 10.1128/mSphere.00548-18

38. Rosado-Franco JJ, Armina-Rodriguez A, Marzan-Rivera N, Burgos AG, Spiliopoulos N, Dorta-Estremera SM, et al. Recombinant Fasciola Hepatica Fatty Acid Binding Protein as a Novel Anti-Inflammatory Biotherapeutic Drug in an Acute Gram-Negative Nonhuman Primate Sepsis Model. Microbiol Spectr (2021) 9(3):e0191021. doi: 10.1128/Spectrum.01910-21

39. Li H, Qiu D, Yang H, Yuan Y, Wu L, Chu L, et al. Therapeutic Efficacy of Excretory-Secretory Products of Trichinella Spiralis Adult Worms on Sepsis- 
Induced Acute Lung Injury in a Mouse Model. Front Cell Infect Microbiol (2021) 11:653843. doi: 10.3389/fcimb.2021.653843

40. Winkler ES, Bailey AL, Kafai NM, Nair S, McCune BT, Yu J, et al. SARS-CoV2 Infection of Human ACE2-Transgenic Mice Causes Severe Lung Inflammation and Impaired Function. Nat Immunol (2020) 21(11):132735. doi: 10.1038/s41590-020-0778-2

41. Hou Y, Zhou Y, Jehi L, Luo Y, Gack MU, Chan TA, et al. Aging-Related Cell Type-Specific Pathophysiologic Immune Responses That Exacerbate Disease Severity in Aged COVID-19 Patients. Aging Cell (2022):e13544. doi: 10.1111/ acel. 13544

42. Chapman PR, Webster R, Giacomin P, Llewellyn S, Becker L, Pearson MS, et al. Vaccination of Human Participants With Attenuated Necator Americanus Hookworm Larvae and Human Challenge in Australia: A Dose-Finding Study and Randomised, Placebo-Controlled, Phase 1 Trial. Lancet Infect Dis (2021) 21(12):1725-36. doi: 10.1016/S1473-3099(21) 00153-5
Conflict of Interest: The authors declare that the research was conducted in the absence of any commercial or financial relationships that could be construed as a potential conflict of interest.

Publisher's Note: All claims expressed in this article are solely those of the authors and do not necessarily represent those of their affiliated organizations, or those of the publisher, the editors and the reviewers. Any product that may be evaluated in this article, or claim that may be made by its manufacturer, is not guaranteed or endorsed by the publisher.

Copyright (๑) 2022 Cai, Mu and McManus. This is an open-access article distributed under the terms of the Creative Commons Attribution License (CC BY). The use, distribution or reproduction in other forums is permitted, provided the original author(s) and the copyright owner(s) are credited and that the original publication in this journal is cited, in accordance with accepted academic practice. No use, distribution or reproduction is permitted which does not comply with these terms. 